\title{
Eine zunächst steile, dann flache Dosiswirkungskurve
}

Fragestellung: Wie steht es um die Wirksamkeit der Diabetesprävention, wenn man alle Arten und ein hohes Ausmaß an körperlicher Aktivität berücksichtigt?

Hintergrund: Wer körperlich aktiv ist, bleibt gesund. Die Aktivität wird in metabolischen Äquivalenten (MET) gemessen als Energie- oder Sauerstoffverbrauch bezogen auf das Körpergewicht und die Dauer. Die Weltgesundheitsorganisation WHO empfiehlt mindestens $600 \mathrm{MET}$ pro Woche, was etwa $150 \mathrm{Mi}-$ nuten zügigem Gehen oder

\section{Originalie}

Kyu H H, Bachman V F, Alexander L T et al. Physical activity and risk of breast cancer, colon cancer, diabetes, ischemic heart disease, and ischemic stroke events: systematic review and dose-response meta-analysis for the Global Burden of Disease Study 2013. BMJ. 2016 Aug 9; 354:13857.
75 Minuten Laufen entspricht. Es gibt aber noch offene Fragen. Einerseits beschäftigen sich Studien vorwiegend mit Freizeitaktivitäten, obwohl quantitativ und qualitativ höchst unterschiedliche körperliche Betätigungen im beruflichen und privaten Bereich hinzukommen. Andererseits ist die WHO-Empfehlung eine Minimalforderung ohne Hin- weis auf das optimale oder sinnvolle Ausmaß der regelmäßigen Aktivitäten.

Patienten und Methoden: Die Autoren suchten weltweit nach Studien, die den Zusammenhang zwischen den mittels MET exakt gemessenen körperlichen Aktivitäten - nicht nur in der Freizeit - und dem Auftreten von fünf Erkrankungen untersuchten. Darunter fanden sich 55 Studien mit 14 Millionen Probandenjahren zum Diabetes in den Jahren zwischen 1999 und 2011.

Ergebnisse: Über das gesamte Spektrum körperlicher Aktivitäten zwischen 0 und 12.000 MET pro Woche zeigte sich: je mehr Aktivität, desto weniger Diabetes. Allerdings lag der überwiegende Teil des Nutzens unterhalb von 3.000 bis 4.000 MET. Im Vergleich zu Personen, die sich körperlich überhaupt nicht betätigten, ging das Diabetesrisiko bei Personen mit bis zu 600 MET um 2\%, bei solchen mit 600 bis 3.600 MET um 19\%, bei 3.600 bis 9.000 MET deutlich geringer und bei über 9.000 MET nur noch um unscheinbare $0,6 \%$ zurück.

Schlussfolgerung: Das Diabetesrisiko kann deutlich stärker gesenkt werden, wenn das Ausmaß der körperlichen Aktivität um ca. fünfmal höher liegt als von der WHO als minimal empfohlen.

\section{- Kommentar von Prof. Dr. med. Heinrich Holzgreve}

\section{Es bleibt noch Zeit zum Verschnaufen}

Die Metaanalyse ist die bisher zuverlässigste Aussage zur Frage, wieviel Sport und körperliche Aktivität sich am besten zur Prävention von Diabetes eignen. Glücklicherweise wird der größte Nutzen in den unteren Bereichen erreicht. Wer sich bisher an die Minimalempfehlung der WHO hielt und mehr erreichen will, muss sich plagen und statt 75 Minuten nun ca. 7,5 Stunden pro Woche laufen. Jenseits dieser Grenze flacht die Dosiswirkungskurve ganz erheblich ab. Ein täglicher Halbmarathon bringt aber nur noch wenig Zusatznutzen und engt anderweitigen Lustgewinn beträchtlich ein.

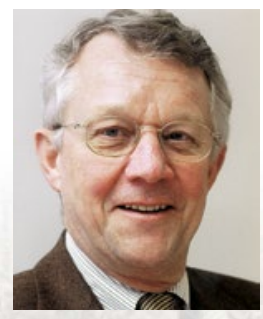

Prof. Dr. med. Heinrich Holzgreve

Internist, Kardiologische Praxis Burgstr. 7, 80331 München heinrich.holzgreve@t-online.de 